\title{
Design of Private Geographycal Information System (GIS) Server for Battlefield Management System (BMS)
}

\author{
Alde Alanda \# , Erwadi Bakar" \\ \# Information Technology Department, Politeknik Negeri Padang, Padang, Indonesia \\ E-mail: aldealanda@gmail.com,erwadib@gmail.com
}

\begin{abstract}
Geographic Information System (GIS) data is needed for model earth surface in 3D simulation for SAR operation to make simulation process as real as possible. Adding integrated GIS data server to simulation system make simulation application user does not need to input and prepare the GIS data manually, by reducing simulation application user task, user can more concentrate on simulation process.In this research the design and implementation of GIS data application that can provide the data needed by a simulation application using existing data on the online map provider. Application designed to display data necessary to carry out the conversion of GIS data to the format used in the simulation. Based on the test resuls of the conversion of GIS data to map format generated simulation has the same texture to the original map. Simulations can be run by using the map conversion and the simulation can run using real map but the level of height accuracy is not optimal.
\end{abstract}

Keywords - GIS, 3D Simulation, Map Conversion

\section{INTRODUCTION}

The rapid advancement in the Information Technology field followed by its application in any field, one of which is the military. On the field, the computer has been widely used in various ways to support existing activities, such as storing important data, satellite imagery, digital mapping, and others. With a very high processing speed in doing the data processing includes image rendering to performing calculations, and therefore one of the most important use is to conduct battle management simulation.

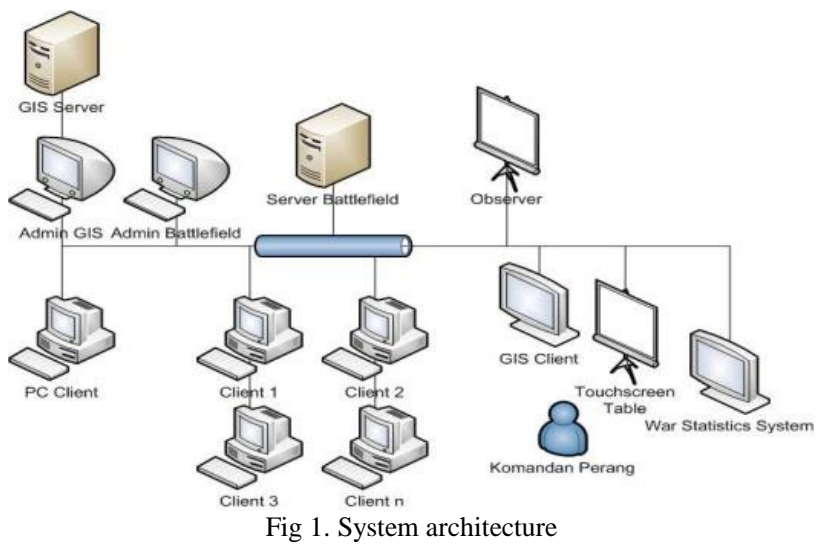

One of the factors that are important in simulating combat is information provided by the map server GIS
(Geographic Information System). GIS is an information system that can display maps and geographic analysis can be used for various purposes. With the use of GIS information, can determine the location of which will be used as a simulation and strategy to be used in combat.

Traditional maps and GIS systems are available in general is a 2D system, it is necessary for conversion to GIS maps can be used in simulation using a 3D system.

\section{BACKGROUND}

Began when England face Germany in World War 2. England have problems of limited facilities and pre-means, both software and hardware (on military personnel ranging from private soldier to officer level, weapons, ammunition, ranpur, aircraft, etc.). Of these problems was born briliyan thinking of the engineers and scientist to solve the problem, namely how to generate wins and profits for a military operation as much as possible but with a minimal sacrifice and pre-given means of limited means. To overcome these problems a new emerging science known as operations research, combined with war-gaming.

System that can be applied to a variety of media, so we can see the various actions the armed forces of developed countries engaged in combat. Where in the fighting which there is movement of thousands of personnel, hundreds of fighter planes, tanks, boats and other battled each other. Well units in all movements of troops, vehicles, aircraft until there 
are thousands of interconnected information. But we do not understand how the flow of information is causing all that happened. Information flows that are more important in the functioning of the military, which now plays an important role in a BMS.

BMS is a management activities in the operational environment based on orders, directives and guidance given by the competent authority. BMS command and control systems for battalion size unit or its equivalent size. BMS provides an overview of a general operation on its users, providing real-time information about the situation in the area of operation and allows the user to prepare and submit a plan [7].

In BMS the important system is Geographic Information System (GIS) that can display a map of the current location of the battle. In the battle of the state of the region plays an important role in decision making eta provided by GIS will assist decisions and strategy while fighting is taking place.

\section{Geographic Information System (GIS)}

Geographic Information System (GIS) is an information system that can display, edit and analyze geographic information referenced coordinates of the earth's surface. The use of GIS is widely used by a variety of scientific fields such as urban planning, land, politics, marketing strategies, cost, and other science simulations [1].

A GIS system should at least have the ability to 1) capture 2) structuring 3) manipulation 4) analysis and 5) presentation [5]. Capturing is the process of spatial data input into the system. Data retrieval can be done manually, semi-automatic and automatic, the output data is a common form of raster and vector. Structuring is a process of spatial information in the data, one of which is to provide coordinate values in the data. Manipulation is the process of editing GIS data such as the change in the value of the feature vector data, transforming data with other systems and other projections. Analysis is the process of calculation of the available data to generate new data is desired, such as the process of making a contour map of a 3D elevation model data. Presentation is the process of displaying the data to the user to provide the desired information. Data displayed may include maps, graphs, tables and other [2].

\section{GIS Application Type}

GIS applications can be divided into GIS desktop and web GIS, GIS is a desktop application that resides on the desktop or on the local computer, GIS is a web application that can be accessed through a web browser [3]. On the web, GIS users, do not need to do preparatory processes data processing to display the desired data in a web browser. While on a desktop GIS data processing required to display the desired map. Behind its simplicity in web GIS user cannot perform the editing process the data because the data is on a server separate from the user's computer, while the desktop GIS users can perform a variety of editing the data and data analysis. Although not able to edit the data available on the web server GIS, web GIS users can add annotations to the data stored in the GIS web server [2].

\section{ANALYSIS AND SYSTEM DESIGN}

Specification systems built into the design of this study is to build a 3D simulation applications using GIS data in order to model the earth's surface close to the original. Applications built simulation is to train tactical battle simulation hostilities commander in combat areas. 3D simulation applications to use the battle as the Glest 3D engine, GIS data obtained from GIS application server capable of retrieving data from a variety of map servers like google, bing and yahoo and the data stored on the server.

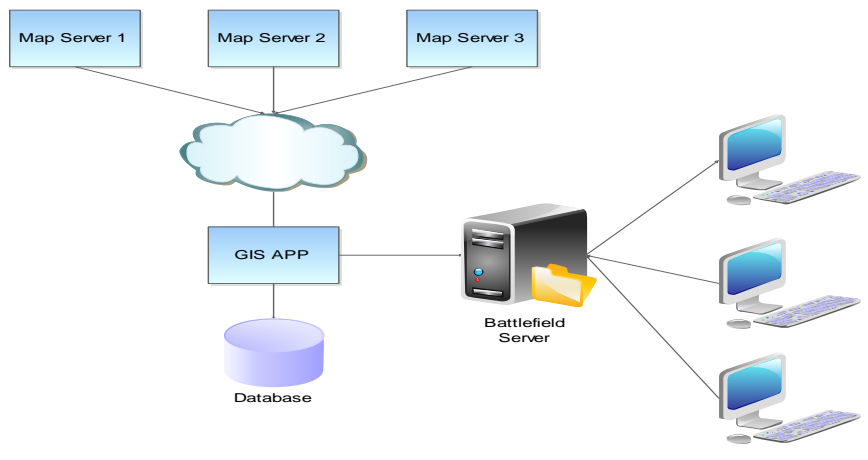

Fig 2. Topology network

\section{Use Case Diagram}

Use case diagrams describe the expected functionality of the server system. Use cases can illustrate the need for the system from the point of view of users, focusing on the process of computerization, describes the relationship between actors and users. In general use case is a pattern of behaviour of the system and the sequence of related transactions performed by an actor. In the GIS data server system for battle simulation there are some functions that will be developed based on the interaction between actors and the system. The actor whose role is admin server GIS data, the master server, admin battlefield, and databases.

\section{Flowchart}

flow chart diagram illustrating the algorithm or process that uses geometric shapes to describe each process and use line arrows to illustrate the flow of processes in a system. By using the entire process flow diagram of a system can be analyzed in stages in accordance with the order process.

\section{Architecture System Design}

Based on system specifications, requirements analysis and system modelling system. System architecture is GIS data server that can retrieve the data from the folder providers and save data on database systems weeks to be cached. the system can also perform conversions folder into a file format that is used by the simulation so that the simulation can be run using data that has been converted 


\section{IMPLEMENTATION}

\section{GIS Implementation}

The implementation of the GIS data server will connect to some online map provider also show the map on 2D and $3 \mathrm{D}$ view.

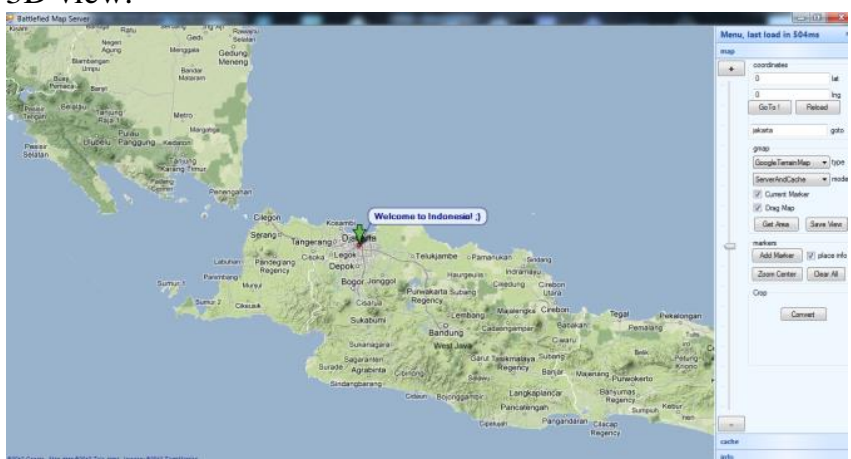

Fig 3. GIS 2D view

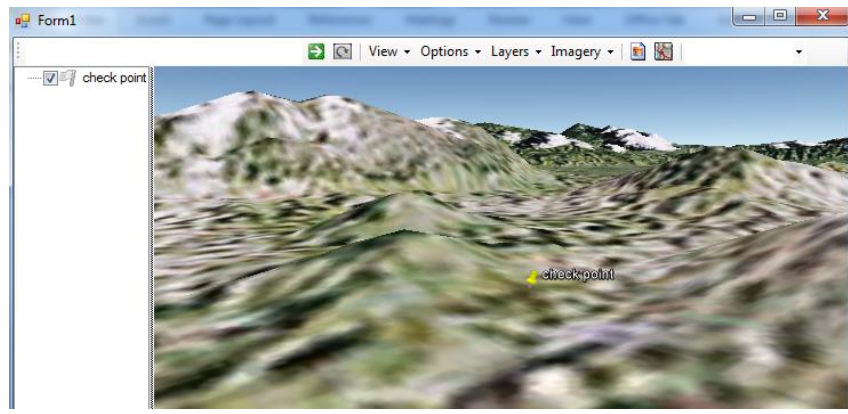

Fig 4. GIS 3D view

\section{Database Implementation}

On the application of GIS database is used to store all the data is automatically accessible online map provider which is then used in the application cache that can be accessed offline.

Provider when accessing the folder and display it on the GIS application will then automatically stored in the database.

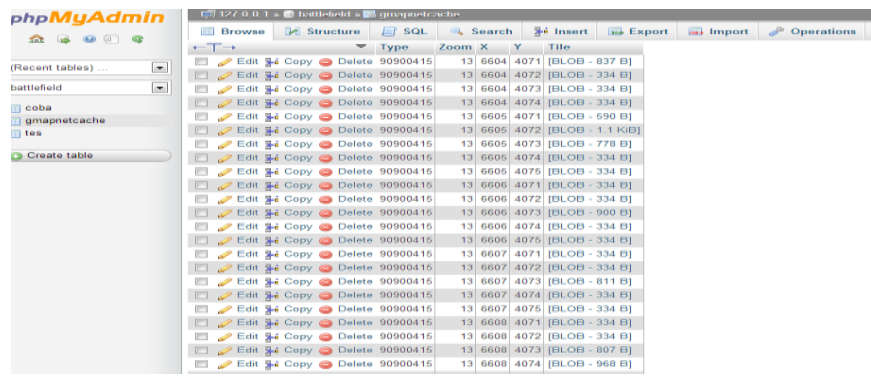

Fig 5. Databases

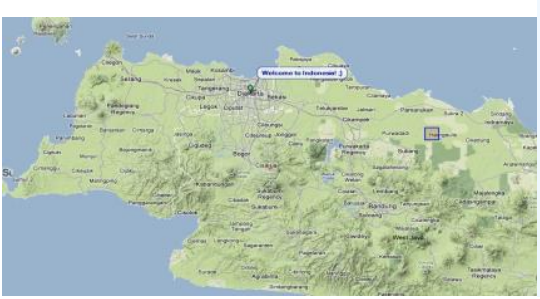

gmap

GoogleTerrainMap type
CacheOnly mode
$\square$ Current Marker
$\square$ Drag Map
Get Area Save View

Fig 6. Database acces

\section{Map Convert}

Conversion folder so that when the simulation is done using the map approaching the real state of the field. Conversion is done from GIS data using PNG format into the data folder simulations using MGM format.

To perform the necessary conversion area that have the same pixel in the simulated map, for example on the area map simulation using the 256x256 pixel area should we take from the GIS has the same size.

Testing performed on maps from GIS data conversion results into folder format used by application simulation.

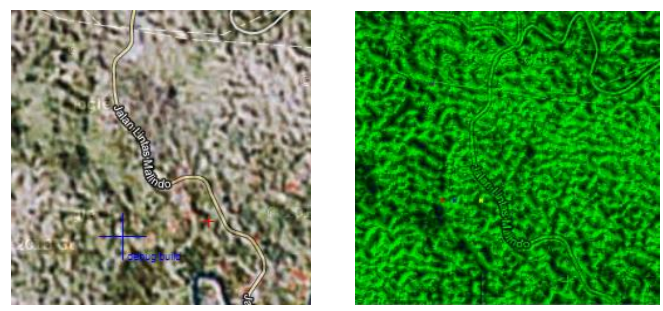

Fig 7. Conversion result

On GIS applications also provide information latitude and longitude coordinates so that the information can be calculated from the distance the actual location on the map using the distance calculation formula based on longitude and latitude.

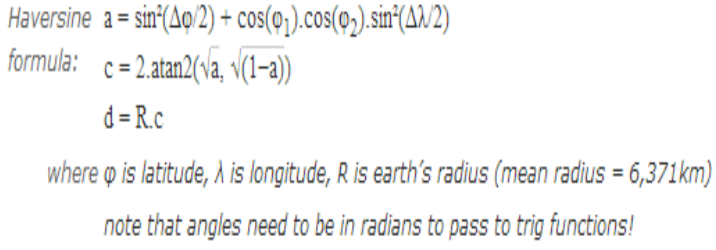

Fig 8 Harversine formula

Location coordinates obtained information entered into the formula harversine so get the distance between two locations that are in the area on the map that is used $256 \times 256$ pixel.

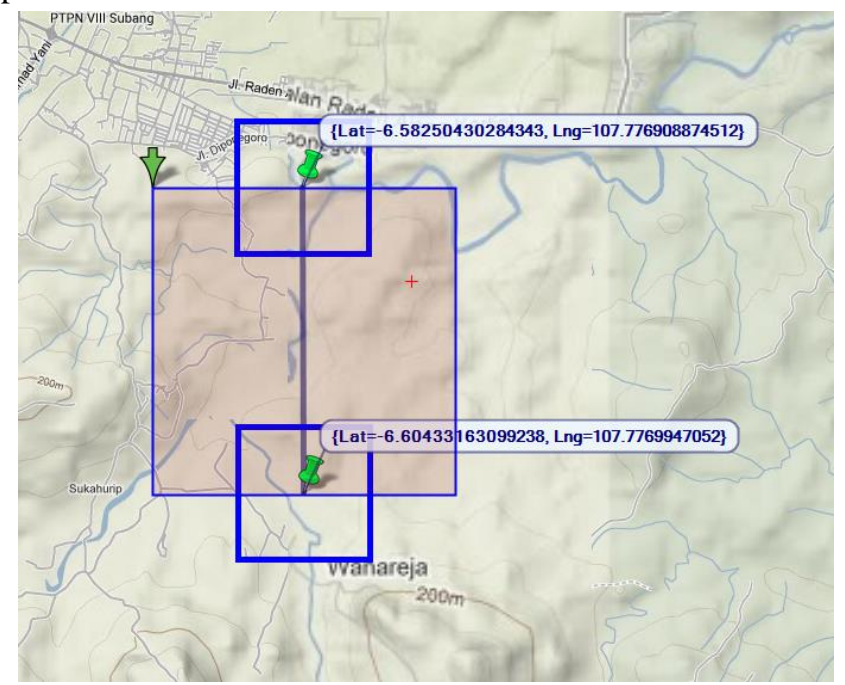

Fig 9 longitude and latitude between 2 points 
Coordinate information can be sought from a distance or length of the actual area used to map $256 \times 256$ with $14 \mathrm{x}$ zoom. The result can be seen in Figure 4.17. For the $256 \times 256$ area has $14 \times$ zoom length or distance of approximately $2 \mathrm{~km}$.

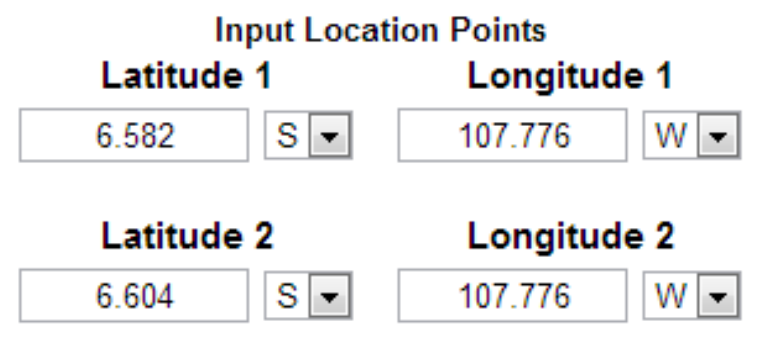

\section{Distance}

(rounded to the nearest whole unit)

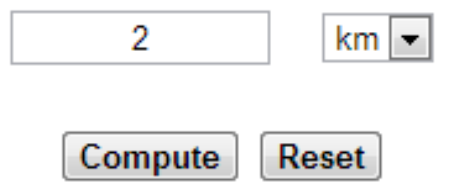

Fig 10 Calculate distance

The map can be running on simulation using map data from GIS. The map on simulation have same texture like real map.

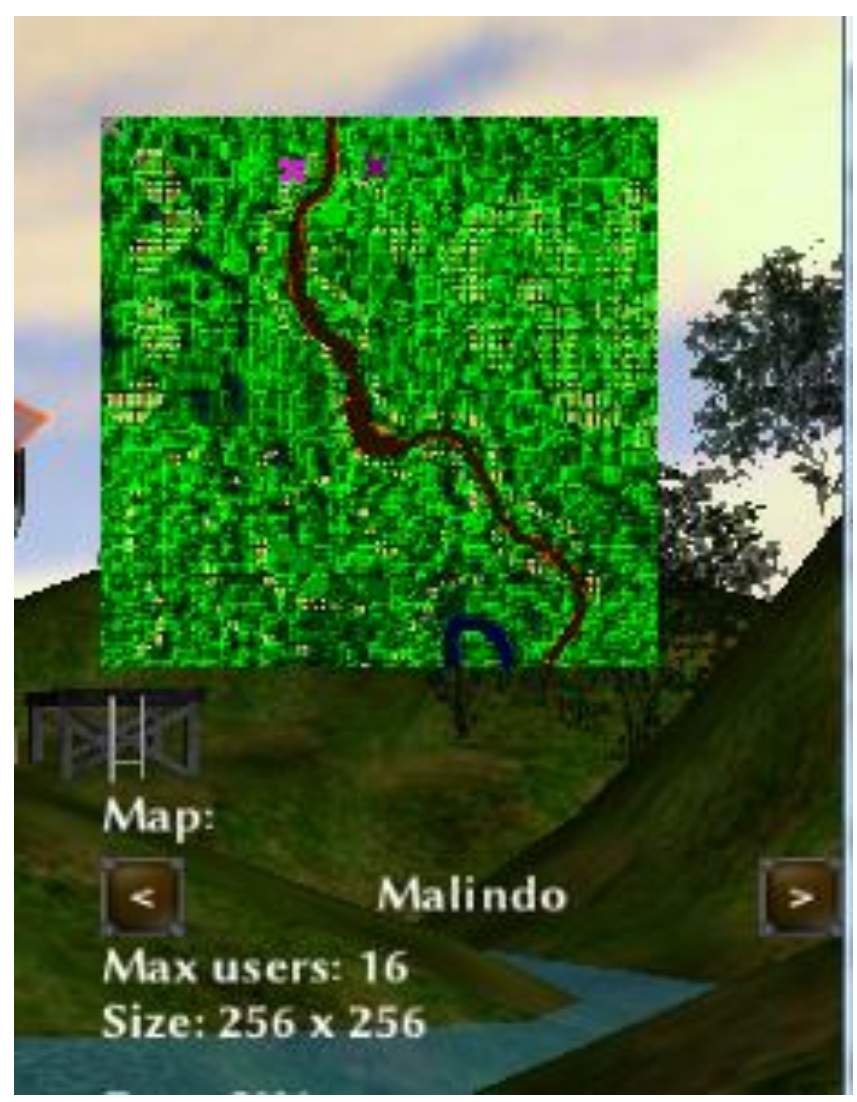

Fig 11. Map preview

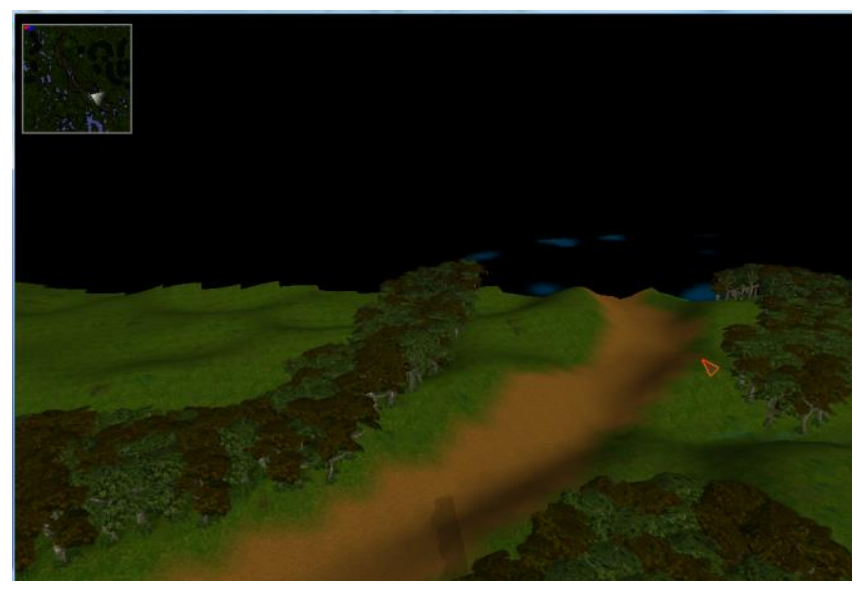

Fig 12. Map running on simulation

\section{CONCLUSION}

Server GIS duty to provide data needed to model the Earth and provide additional data. Required fields are, image and likeness earth vector data have additional information such as city name, street and river maps and displays in $2 \mathrm{D}$ and $3 \mathrm{D}$.

GIS applications are able to connect to a variety of online map providers so as to display the image data in the form of surface and send 2D and 3D data from GIS applications to a database that can be accessed by other applications such as simulation applications.

The conversion was successful simulation applications to run on texture similarity level $90 \%$ the same as the original map, but in terms of the height map accuracy is still not optimal.

\section{REFERENCES}

[1] Murai, Shunji,GIS Workbook Vol I, University of Tokyo, Tokyo.2006.

[2] Prahasta, Eddy.,Konsep-Konsep Dasar Sistem Informasi Geografis, Bandung, CV. Informatika.2007.

[3] Sherman, Garry E, Desktop GIS, The Pragmatic Bookshelf, Dallas, 12-22,

[4] D. G. Bell et al. (2, NASA World Wind: Opensource GIS for Mission Operations, IEEE Aerospace Conference, 1-9.

[5] Chrisman, Nicholas R, What Does GIS Mean, , University of Washington,2000.

[6] Vertegaal, M.Sc., Merel, "Delelopment of battlefield management system : how to use the user", The Netherlands.

[7] Lickteig, Carl W., "Design Guidelines and Functional Specifications for Simulation of the Battlefield Management System's (BMS) User Interface”, ARI Field Unit, July, 1988.

[8] Burdick, Howard E,Digital Imaging Theory And Applications, McGraw-Hill Companies,Inc, New York,1998. 